\title{
Damage Detection System with Sub-microsecond Resolution
}

E. Udd, J. Benterou

February 14, 2008

SPIE Smart Structures and Materials \& Non-destructive Health Monitoring San Diego, CA, United States March 9, 2008 through March 13, 2008 
This document was prepared as an account of work sponsored by an agency of the United States government. Neither the United States government nor Lawrence Livermore National Security, LLC, nor any of their employees makes any warranty, expressed or implied, or assumes any legal liability or responsibility for the accuracy, completeness, or usefulness of any information, apparatus, product, or process disclosed, or represents that its use would not infringe privately owned rights. Reference herein to any specific commercial product, process, or service by trade name, trademark, manufacturer, or otherwise does not necessarily constitute or imply its endorsement, recommendation, or favoring by the United States government or Lawrence Livermore National Security, LLC. The views and opinions of authors expressed herein do not necessarily state or reflect those of the United States government or Lawrence Livermore National Security, LLC, and shall not be used for advertising or product endorsement purposes. 


\title{
Damage Detection System with Sub-microsecond Resolution
}

\author{
Eric $\operatorname{Udd}^{1}$ and Jerry Benterou ${ }^{2}$ \\ ${ }^{1}$ Columbia Gorge Research, LLC, 2555 NE $205^{\text {th }}$ Avenue, Fairview, Oregon 97024 \\ ${ }^{2}$ Lawrence Livermore National Laboratory, 7000 East Avenue, Livermore, California 94550
}

\begin{abstract}
Fiber optic grating sensors have been used to measure multi-dimensional strain, pressure, temperature, corrosion and moisture. This paper presents a method of using fiber grating sensors to measure the position and velocity of a very fast event associated with a blast wave. A chirped fiber grating of $50 \mathrm{~mm}$ length is placed in a highly energetic material. The action of the shock wave is to destroy the fiber grating as it propagates along it. By using a spectral filter such as a chirped fiber grating in combination with high speed detectors the position and velocity of the shock wave may be determined. A layout of a system used to experimentally verify this technique is described and results presented for two different highly energetic materials.
\end{abstract}

Keywords: fiber optic, fiber Bragg grating, fast, detection

\section{INTRODUCTION}

Fiber Bragg grating sensors have been used to monitor high speed events to support applications such as measurements of strain in structures such as utility poles, bridges and elevated freeway ramps. Higher speed systems have been used to support ultrasonic systems with detection speeds of a few hundred kilohertz to a few MHz.

In order to monitor detonation waves associated with very highly energetic materials such as high explosives and rocket propellants it is desirable to be able to measure events with a system that can respond at GHz speed. It was also necessary to establish a system that was capable of making highly precise measurements over distances on the order of at least a few $\mathrm{cm}$ to support initial proof of principle testing. Early testing was done using conventional fiber gratings in combination with chirped fiber gratings to provide a spectral filter. The success of these tests led to the usage of chirped fiber gratings as sensors to enable the prospect of more complete and quantitative in-situ diagnostics to be embedded directly within an explosive charge.

Improvements include establishing techniques that enable chirped fiber Bragg grating (FBG) sensors to have welldefined edges allowing accurate measurements of the physical length of the sensor. Additional improvements include developing techniques for drilling extremely small diameter holes in high explosives which would allow the insertion of FBG sensors into the bulk high explosive which will cause minimal perturbation to the detonation wave being measured. FBG sensors can then be placed inside high explosives and propellants to measure with high accuracy, the detonation velocity and physical parameters associated with very high-speed events.

\section{APPLICATIONS OF IN-SITU DETONATION VELOCITY SENSORS}

There have been two types of metrology techniques, which continuously measure the detonation velocity of an explosive in the run-up to full order detonation. The first is an older technique based on microwave interferometry where a cavity is set up in a microwave waveguide filled with the explosive and interference from the reflection off of the plasma created at the detonation wave front forms a beat signal with the reference beam to continuously measure the detonation velocity acceleration. Limitations are the relatively small return signal, the large waveguide (and larger explosive sample) needed, and the conformance to a relatively linear waveguide detonation velocity measurement. The 
velocity also needs to be de-convolved from the beat frequency of the microwave interference fringes from the detonation. A newer, more recently developed technique involves embedding plastic liquid-filled optical fibers within in an explosive volume where velocity is measured directly as Doppler shifted light reflected off the shock wavefront within a liquid inside a section of polyethylene tubing. As this technique uses current Fabry-Perot technology to measure the Doppler shift of the light, it provides a continuous measurement rather than a series of average velocities between measurement pins. This paper derives from some work originally developed using liquid light guides filled with a material which exhibits a significant jump in refractive index for a jump in pressure at the shock wavefront and applies it in a new direction using very much smaller telecommunication grade optical fibers. The advantage of telecom grade fiber is a very small diameter (125 microns typically, with a polyimide coating of 5 to 10 microns for protections) that has a minimal effect on the detonation behavior of non-ideal explosives with large failure diameters. Rather than look at the apparent Doppler shifted light reflected at the interface of the shock front - we literally print a chirped refractive index grating (commonly referred as a fiber Bragg grating) within a linear section of the fiber. A long exposure of ultraviolet radiation through a phase mask makes the core of the fiber selectively reflect back light over a specified passband. An extended fiber optic light source, specified to extend over the defined passband of the fiber Bragg grating, provides illumination on the fiber Bragg grating. Because the grating is printed onto the physical fiber core, as the fiber is destroyed, the reflected light disappears, reducing the signal from the photodetector. The total light measured by a photodetector is a convolution integral of the spectral reflection function of the fiber Bragg grating, the spectral emission function of the 1.55 micron light source, and the spectral response function of the photodetector.

\section{MEASURING DETONATION VELOCITY USING CHIRPED FIBER GRATINGS}

Knowing the behavior of ideal high explosives during the dynamic transition from early low-order detonation to the full-order detonation is critical in improving hydrocode models of the initiation and detonation process. For modern, non-ideal insensitive explosives being developed today, these transitions scale to longer times and distances and lead to changes in failure diameters. Experimental techniques that measure a continuous change in detonation velocity provide insight into this dynamic regime of initiation physics. Continuous velocity measurements are significantly more accurate in determining this run-up in velocity relative to single point measurements (such as ionization pins or piezoelectric shock pins), which yield only the average velocity measurement between the individual pin placement points.

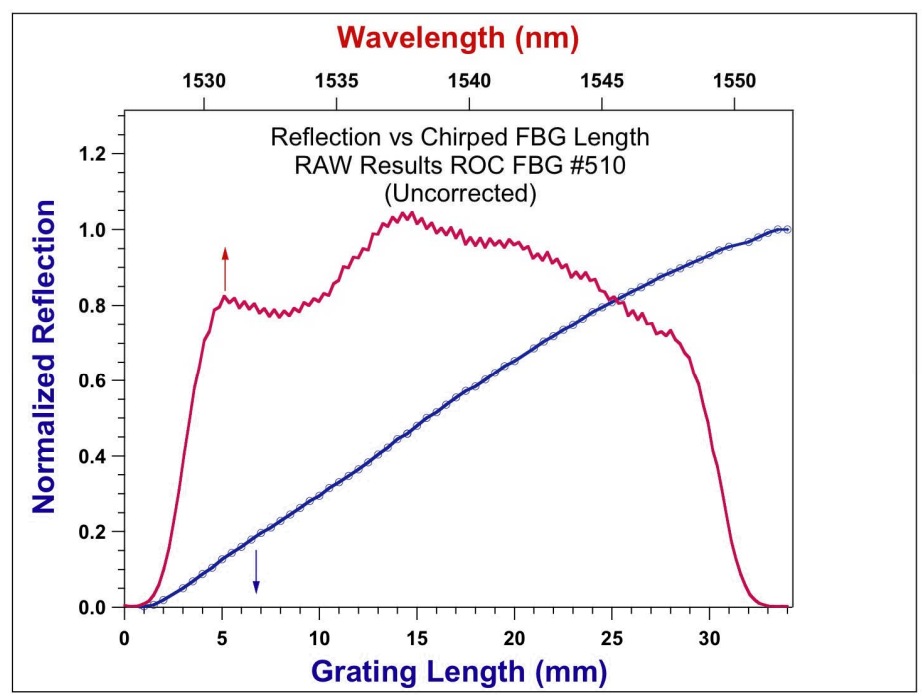

Figure 1.

Spectrum and bulk reflection of a $34 \mathrm{~mm}$ chirped FBG that was laser-cut using a femtosecond laser. The "bumps" on the FBG spectrum come from the amplitude variations of the ASE illumination. 
Figure 1 illustrates this principle. A $34 \mathrm{~mm}$ chirped fiber grating was physically cut back in $0.5 \mathrm{~mm}$ increments through its entire length, simulating the shortening of the FBG caused by the propagating detonation. By monitoring the changes in the spectral reflection band, the length of the chirped fiber grating may be determined continuously. This is the same thing one would expect to see when an FBG is being consumed by an advancing detonation wave.

\section{IN-SITU DETONATION VELOCITY MEASUREMENT EXPERIMENTS}

The overall layout of the test system is shown in Figure 2. Here a broad band ASE light source, which in this case is a gain flattened $1550 \mathrm{~nm}$ erbium fiber amplified stimulated emission light source pumped by a $980 \mathrm{~nm}$ laser diode is used to inject light into a 50/50 coupler which has one of its output fiber legs attached to a chirped fiber grating that is placed in a high explosive. When the detonation occurs, a portion of the chirped fiber grating is destroyed and the spectral reflectance decreases. The signal that is back reflected from the chirped fiber grating is directed into a second 50/50 coupler. One output leg of this second coupler is directed into a reference detector that monitors the changes in the spectral reflection directly. The second output fiber leg is directed into an optical fiber that contains a second chirped fiber grating that has a spectral reflection that is the same as the chirped fiber grating that is placed in the high explosive. The reflection from this second chirped fiber grating is then directed back into the second 50/50 coupler and a portion of this reflection is then directed to a second detector used to monitor this reflected signal. Since the chirped fiber grating, used as the sensor, and the second chirped fiber grating, used as the reflector, overlay spectrally, light that is associated with the blast that is not in the spectral band of the chirped fiber grating sensor is filtered from the output detector. By comparing the reference and reflected signals on the detectors, light induced by the blast wave in the detection band can be monitored and subtracted from the overall signal.

To demonstrate the viability of this technique, we constructed an apparatus to launch light into a sensor fiber with an embedded phase grating, then placed this sensor fiber in a series of explosive volumes and recorded the integrated decrease in light from the chirped FBG as it was destroyed. Two detonation run-up experiments were conducted at the High Explosive Applications Facility at LLNL in June 2007 using Nitromethane as the sample liquid explosive in one experiment and pressed pellets of PBX-9502, a modern insensitive explosive, in the other. 


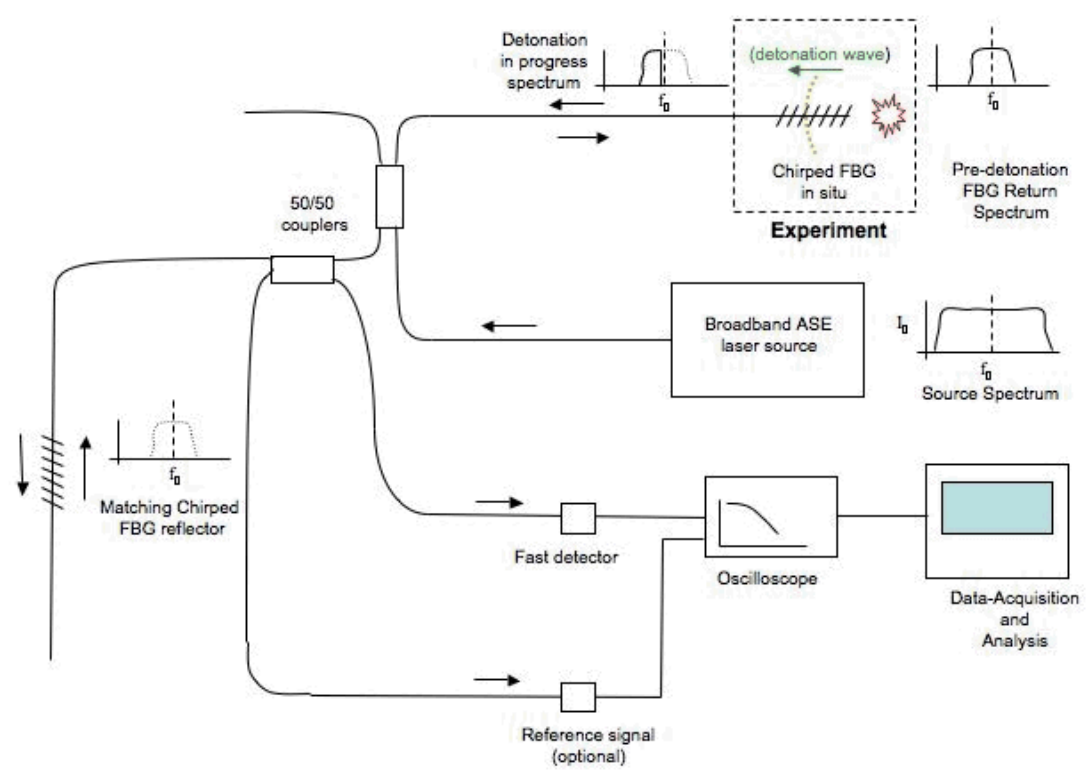

Figure 2.

Fiber Bragg grating detonation velocity sensor system block diagram.

\section{MEASUREMENT OF DETONATION VELOCITY INSIDE NITROMETHANE}

The first test detonation involved placement of an optical fiber with a chirped FBG with a length of approximately 36 $\mathrm{mm}$ into a cylindrical container of nitromethane as shown in Figure 3. One end of the chirped fiber grating was placed at the bottom of the cylinder adjacent to a booster explosive (Comp-B).

The test results from the first test shot are shown in Figure 4. Both the reference and reflected signal detectors associated with Figure 3 show the chirped fiber grating being destroyed at the same rate. This would indicate that the light associated with the nitromethane blast is not affecting the output reference signal in a significant way. The manufacturer of the chirped fiber grating was originally targeting an overall physical length of $50 \mathrm{~mm}$. However when laser cut back tests were performed on a second identical " $50 \mathrm{~mm}$ " chirped FBG, the spectrum did not change until it was cut back approximately $14 \mathrm{~mm}$ from the expected edge of the grating. This indicates that the physical length associated with the spectral band of the cut back grating \#509 was about 35 or $36 \mathrm{~mm}$. This matches up very well with the velocity associated with the pin timing used to support the first detonation test.

This unexpected result (i.e., discovering that the physical length of the FBG was not the same as the manufacturer's specification) emphasizes a key principle. In order to derive an accurate detonation velocity measurement, one needs to first know the true physical length of the FBG. 

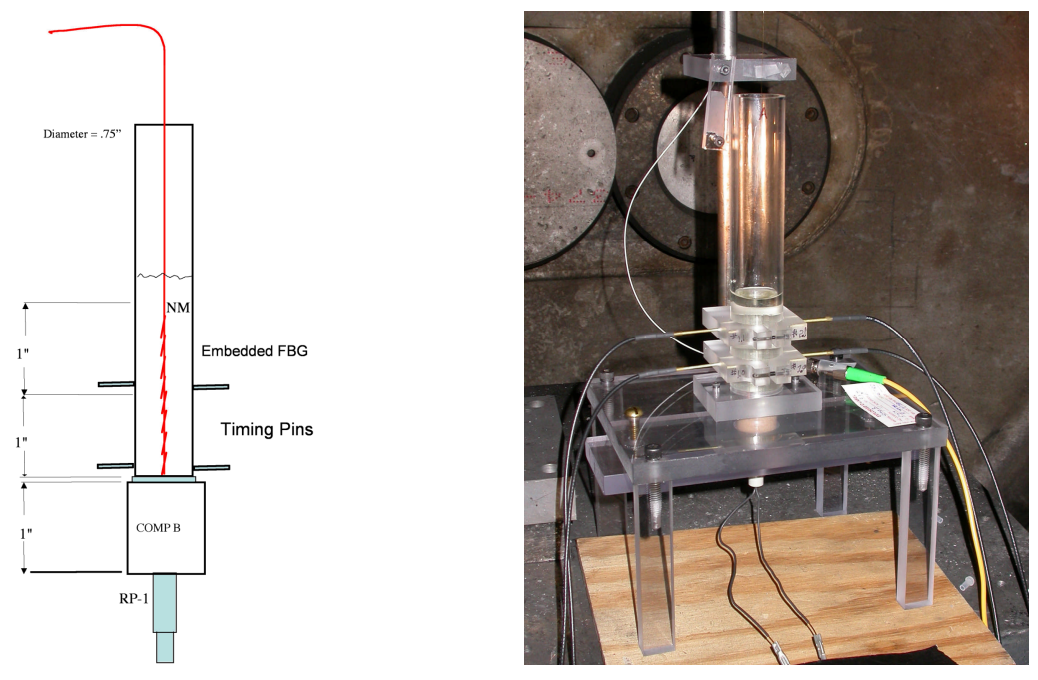

Figure 3.

Nitromethane detonation test configuration. A 36 mm FBG was embedded into the liquid nitromethane.

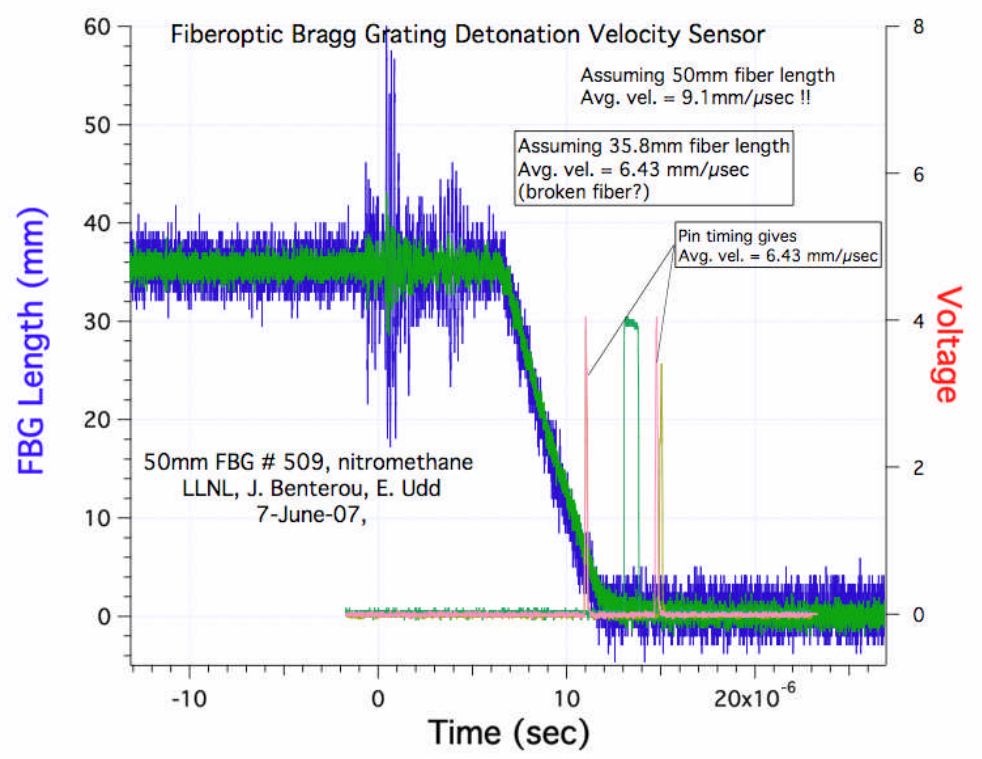

Figure 4.

In-situ velocity measurement of detonating nitromethane. Noise in signal comes from fireset EMP and low signal level from detectors.

\section{MEASUREMENT OF DETONATION VELOCITY INSIDE PBX-9502}

The second test set was supported using a cylinder packed with four $25.4 \mathrm{~mm}$ diameter PBX-9502 pellets $25.4 \mathrm{~mm}$ long. The effective spectral "length" of the second " $100 \mathrm{~mm}$ " chirped fiber grating was approximately $62 \mathrm{~mm}$ in length (measured by laser cut back testing of a similar " $100 \mathrm{~mm}$ " FBG). The $62 \mathrm{~mm}$ fiber grating was inserted into the center of cylinder filled with PBX-9502 pellets as illustrated by Figure 5. The edge of one side of the $62 \mathrm{~mm}$ chirped fiber grating was placed near the Comp-B booster and provided coverage through nearly three sections of pellets during the second blast event. 


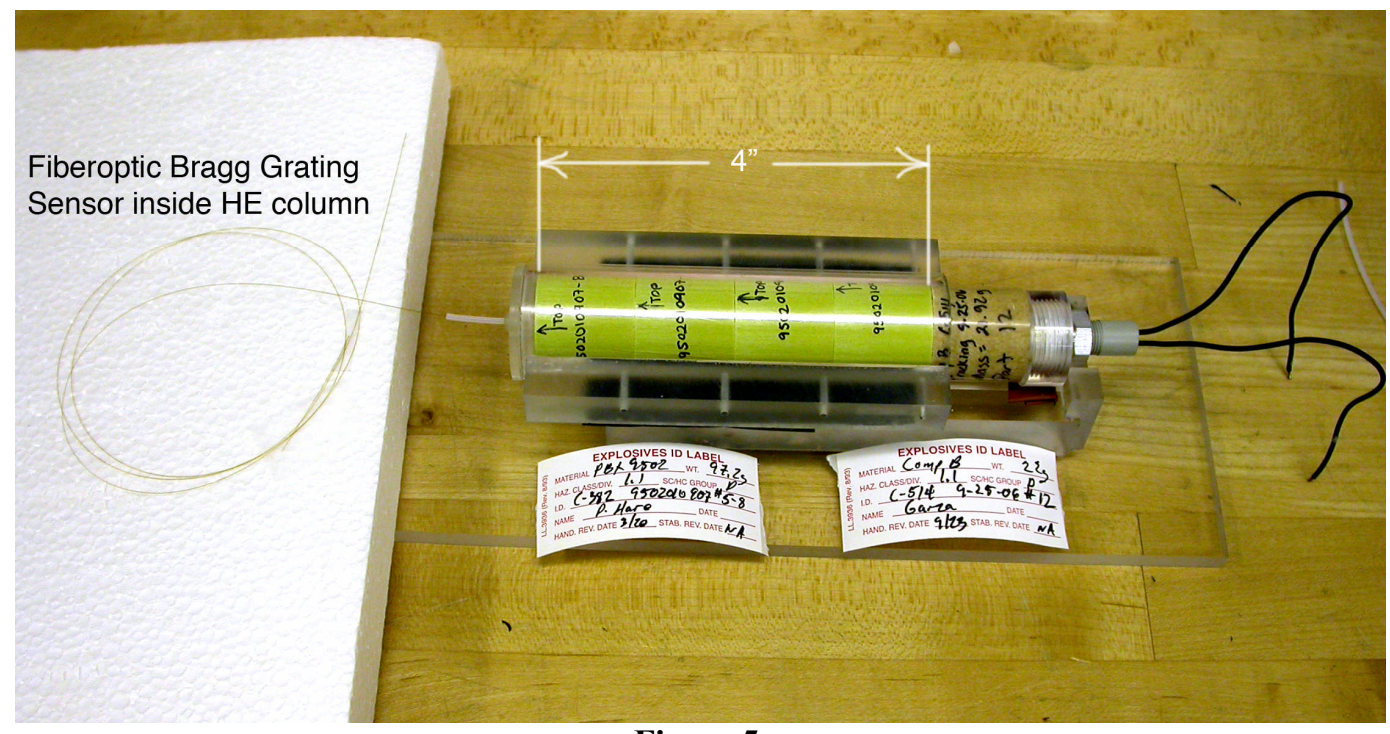

Figure 5.

Experimental setup for PBX-9502 in-situ velocity test. Fiber-optic Bragg grating sensor is embedded in the high explosive.

Figure 6 shows the test results for the second shot that was conducted in a larger test tank. The results appear similar to the first test although there is additional noise associated with the last third of the slope associated with the destruction of the chirped fiber grating. This may be due to electrical pickup by the circuitry associated with the optical detectors. If this is the case, better shielding and isolation could be applied to eliminate this noise source. An electrical transient on the optical detector waveform is seen and correlates to the high-current capacitor discharge unit firing and bursting the bridgewire in the RP-1 detonator.

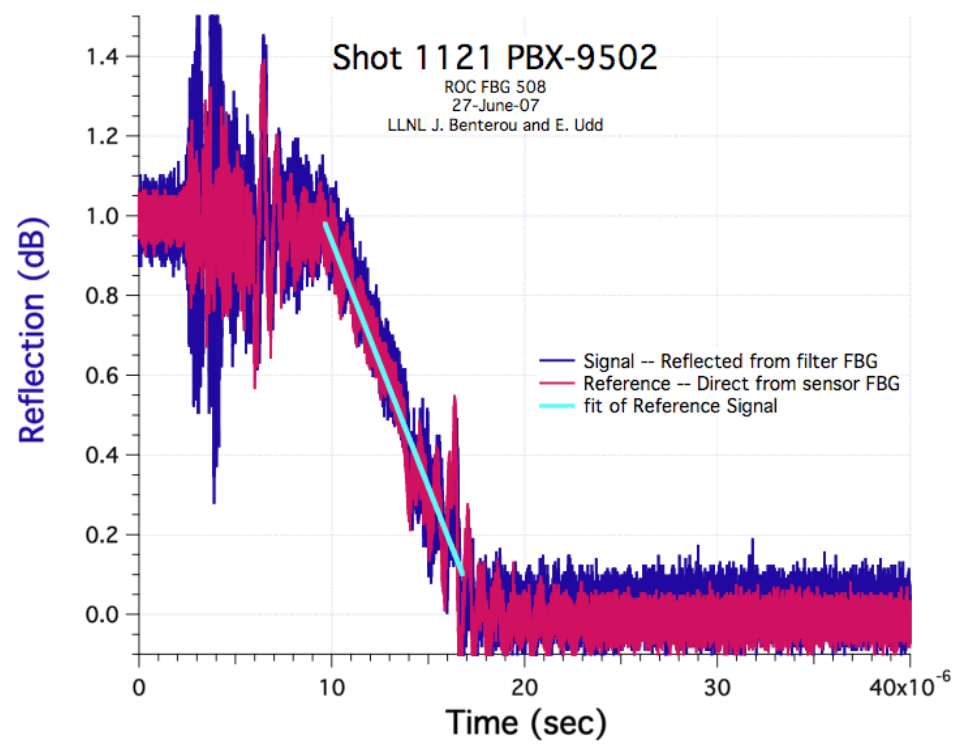

Figure 6.

Preliminary results of in-situ velocity measurement of detonating PBX-9502. Assuming the true physical length of the FBG is $62 \mathrm{~mm}$, the velocity derived from this record is approximately $7.7 \mathrm{~mm} / \mathrm{microsecond}$. 
Also, the addition of a fast trans-impedance amplifier stage after the detector stage would boost the current signal well above the baseline noise of the digitizing oscilloscope. Since the fiber grating sensor system is electrically isolated except for the electro-optic component circuitry in the control room (light source driver and detector support circuits), the prospects of reducing this source of noise are very good.

\section{DETERMINATION OF THE PRECISION OF VELOCITY MEASUREMENTS}

During the course of performing these tests, it became evident that the chirped FBG specified at $100 \mathrm{~mm}$ actually had a physical length of approximately $62 \mathrm{~mm}$. This was determined by using two sets of gratings on a physical cut-back test on the 100-mm and 50-mm chirped fiber gratings. This was done by laying out the fiber gratings in a straight line and then physically cutting them back with a femtosecond laser by increments of $0.5 \mathrm{~mm}$ of fiber until the spectral band of the chirped fiber grating changed in a measurable manner. The spectrometer used to support these tests was an Ibsen IMON 400 that can be operated at a sample bandwidth of $200 \mathrm{~Hz}$ over the 1520 to $1580 \mathrm{~nm}$ spectral band. This spatial calibration was very useful in providing real time feedback during the cut back tests.

\section{ANALYSIS OF IN-SITU DETONATION VELOCITY MEASUREMENTS}

Besides detonation experiments, several of the fiber gratings as described above were cut back from the longer wavelength end until a clear transition in response was observed. This allowed an unambiguous starting point for the chirped fiber grating sensor position. Plotting the response via a cut back test also allowed the overall position and effective length of the chirped fiber grating to be established. For example, a so-called "50 mm" FBG was lasertrimmed at $0.5 \mathrm{~mm}$ increments along its entire reflection band pass shown in figure 7 . It was quickly discovered that the true physical length of the FGB was $34 \mathrm{~mm}$, not $50 \mathrm{~mm}$ as specified by the manufacturer.

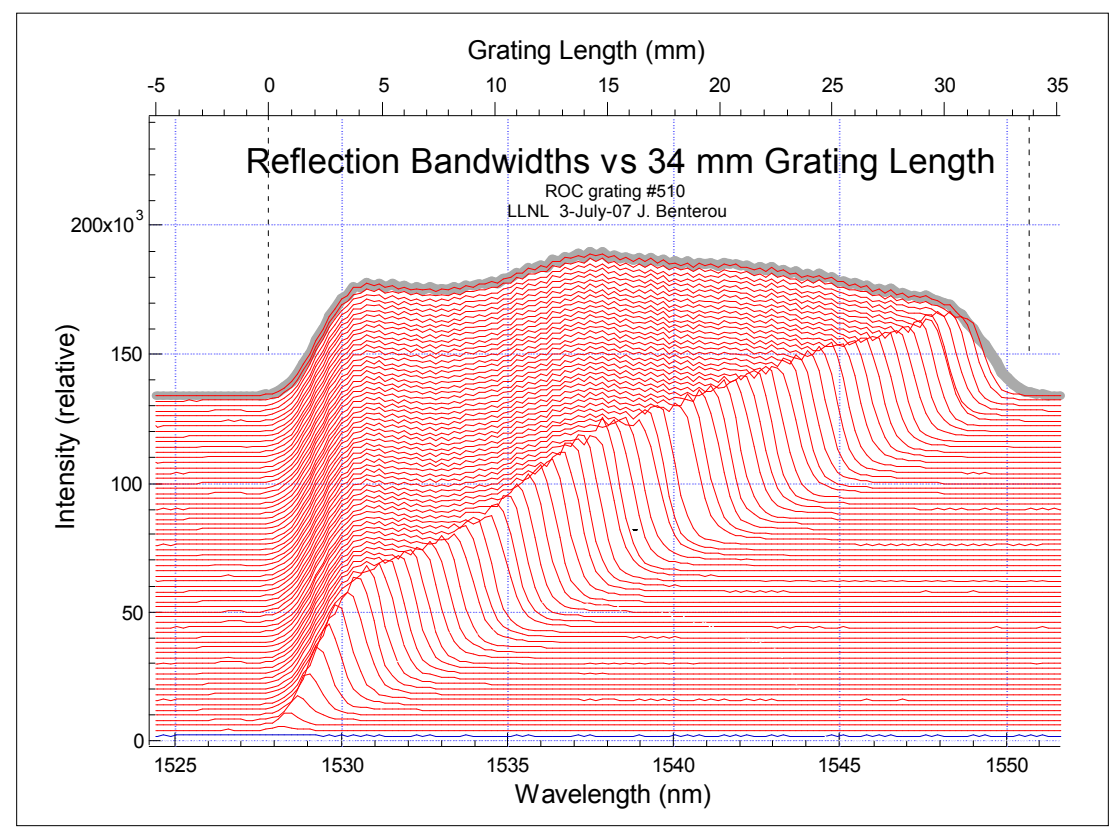

Figure 7.

Waterfall plot of grating spectra vs. FBG length.

There is very good agreement between the raw data from the laser cutback test, and a numerical integration of the 34 mm FBG reflection bandwidth (see figure 8). This implies a procedure in which the physical length of the FBG is 
measured and the optical reflection bandwidth is recorded prior to the detonation. Knowing the physical length and the reflection bandwidth of the FBG sensor is required in order to derive the position vs. time history of the detonation. The velocity of detonation can be calculated by simply taking the derivative of the FBG length vs. time data. Since the data recorded is on a digital oscilloscope, this continuous measurement can yield an ASCII file containing the continuous record of the detonation velocity inside the explosive being studied.

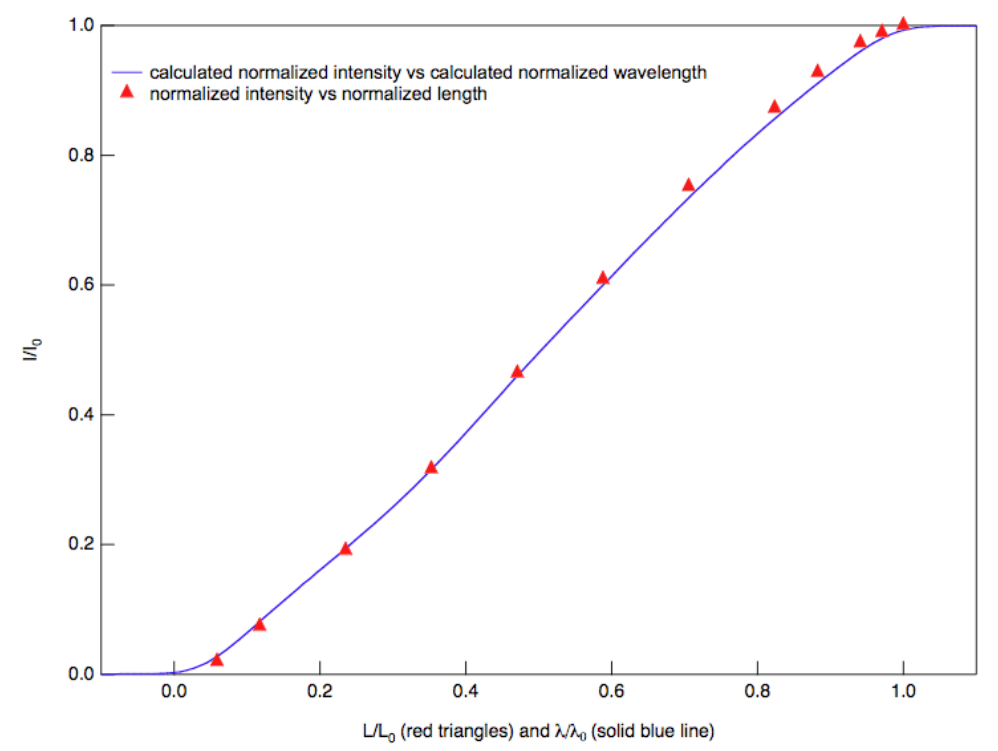

Figure 8.

Actual data (red triangles) from FBG reflection laser cut-back test shows good agreement with the numerical integration of the chirped FBG bandwidth.

\section{IMPROVING DETONATION VELOCITY MEASUREMENT ACCURACY}

Since it is essential to know the absolute physical length of the FBG and its location inside the high explosive or propellant before detonation, a reliable method to pre-measure the FBG length must be developed. Dispersion measurements using a tunable diode laser can be used to resolve FBG lengths to 300 microns. Therefore, knowing the FBG length to this accuracy promises that $50 \mathrm{~mm}$ FBG velocity measurements could be accurate to less than $1 \%$. Also, the ASE source amplitude variations can be cancelled by dividing the raw oscilloscope signal by the normalized transfer function.

By exposing light of a greater bandwidth than the chirped fiber Bragg grating down the fiber through a circulator (or $2 \times 2$ coupler), the integral of the light reflected back under the bandwidth of the chirped fiber Bragg grating is the total light coming back from the circulator into a detector. Using a high-speed InGaAs/PIN photodetector and assuming a relatively flat-topped chirped passband for reflection and a spectrally flat light source, a linear reduction of reflected light back to the detector indicates that the shock wave is linearly destroying the chirped fiber Bragg grating. This optical signal reduction measures the integrated progress of the destruction of the printed grating by the detonation shock front.

Accurate measurement of the FBG length and accurate FBG placement will result in improved velocity and position information. The prospect of further improvements in this diagnostic to yield reliable, accurate and repeatable in-situ detonation velocity measurements is quite achievable. 


\section{DRILLING SMALL HOLES IN ENERGETIC MATERIALS}

In order to accurately measure the in-situ continuous detonation velocity, the FBG sensor must be physically inserted into the high explosive or propellant and the sensor location must be well known with respect to the surrounding energetic material. This requires the development of methods to safely and inexpensively make small diameter holes in the energetic materials. One approach is to use a femtosecond laser beam to drill 100 to 200 microns diameter holes of medium depth up to $8 \mathrm{~mm}$ deep. The femtosecond laser drilling is performed inside an explosive containment tank and is controlled remotely by the operator. For deeper holes of depths from 10 to 100 s of millimeters deep, rotating drill bits can be used, provided that safety considerations (friction, heat, shock, pressure) are taken into account. For example, machining and hole drilling of high explosives at Lawrence Livermore National Laboratory is done routinely as a remote-controlled operation inside explosive containment tanks.

\section{ACKNOWLEDGEMENTS}

Portions of this paper were drawn from:

"In-Situ Continuous Detonation Velocity Measurements Using Fiber-optic Bragg Grating Sensors", (UCRL-PROC233137), by Jerry BENTEROU, Eric UDD, Paul WILKINS, Frank ROESKE, Ed ROOS, and Damon JACKSON; Proceedings of the $34^{\text {th }}$ International Pyrotechniques Seminar, Vol. 1, pp. 306, Beaune, France (2007)

\section{OTHER REFERENCES}

1. Udd, Eric and Calvert, Sean; "Fiber Grating Pressure Wave Speed Measurement System", US Patent Application 11/071,278 filed March 3, 2005 (partially based on US Provisional Patent Application 60/552,846 filed March 12, 2004)

2. Martin, Guy; Trépanier, François; "Fiber Bragg Gratings: Manufacturing Techniques"; The Photonics Handbook 2007, $53^{\text {rd }}$ International Edition; Laurin Publishing, 2007.

3. Kersey, A. D., et al.; "Fiber Grating Sensors", IEEE Journal of Lightwave Technology, Vol. 15, pp. 1442-1463, 1997.

4. Cheng, van Bree; "Fiber-optic sensors in explosions and detonation experiments", SPIE Newsroom, 2 Feb. 2007.

5. Prinse, W. C.; "Development of fiber optic sensors at TNO for explosion and shock wave measurements", Proceedings of the SPIE 4183, pp. 748-758, 2000.

6. Prinse, W. C.; "High-speed velocity measurements on an EFI-system", Proceedings of the SPIE 6279, 1 Jan. 2006.

This work was performed in 2007 and 2008 under the auspices of the U. S. Department of Energy by the Lawrence Livermore National Laboratory under contracts W-7405-ENG-48 and DE-AC52-07NA27344. 